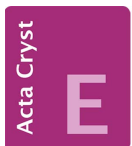

CRYSTALLOGRAPHIC COMMUNICATIONS

ISSN 2056-9890

Received 22 November 2015

Accepted 14 December 2015

Edited by G. Smith, Queensland University of Technology, Australia

Keywords: coordination polymers; crystal structure; lanthanide; luminescence; terbium(III)

CCDC reference: 1436132

Supporting information: this article has supporting information at journals.iucr.org/e

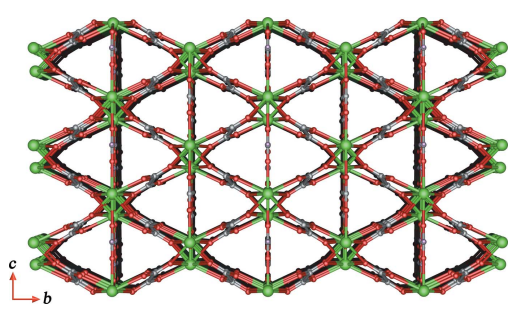

OPEN $\odot$ ACCESS

\section{Crystal structure of a mixed-ligand terbium(III) coordination polymer containing oxalate and formate ligands, having a three-dimensional fcu topology}

\author{
Chainok Kittipong, ${ }^{a *}$ Phailyn Khemthong, ${ }^{b}$ Filip Kielar ${ }^{b}$ and Yan Zhou ${ }^{c}$
}

aDepartment of Physics, Faculty of Science and Technology, Thammasat University, Khlong Luang, Pathum Thani, 12120, Thailand, 'b Department of Chemistry, Faculty of Science, Naresuan University, Muang, Phitsanulok, 65000, Thailand, and ${ }^{\mathrm{c}}$ Department of Chemistry, The Hong Kong University of Science and Technology, Clear Water Bay, Kowloon, Hong Kong. *Correspondence e-mail: kc@tu.ac.th

The title compound, poly[( $\mu_{3}$-formato $)\left(\mu_{4}\right.$-oxalato $)$ terbium(III)], $\left[\mathrm{Tb}\left(\mathrm{CHO}_{2}\right)\right.$ $\left.\left(\mathrm{C}_{2} \mathrm{O}_{4}\right)\right]_{n}$, is a three-dimensional coordination polymer, and is isotypic with the $\mathrm{La}^{\mathrm{III}}, \mathrm{Ce}^{\mathrm{III}}$ and $\mathrm{Sm}^{\mathrm{III}}$ analogues. The asymmetric unit contains one $\mathrm{Tb}^{\mathrm{III}}$ ion, one formate anion $\left(\mathrm{CHO}_{2}{ }^{-}\right)$and half of an oxalate anion $\left(\mathrm{C}_{2} \mathrm{O}_{4}{ }^{2-}\right)$, the latter being completed by application of inversion symmetry. The $\mathrm{Tb}^{\mathrm{III}}$ ion is ninecoordinated in a distorted tricapped trigonal-prismatic manner by two chelating carboxylate groups from two $\mathrm{C}_{2} \mathrm{O}_{4}{ }^{2-}$ ligands, two carboxylate oxygen atoms from another two $\mathrm{C}_{2} \mathrm{O}_{4}{ }^{2-}$ ligands and three oxygen atoms from three $\mathrm{CHO}_{2}{ }^{-}$ ligands, with the $\mathrm{Tb}-\mathrm{O}$ bond lengths and the $\mathrm{O}-\mathrm{Tb}-\mathrm{O}$ bond angles ranging from 2.4165 (19) to 2.478 (3) $\AA$ and 64.53 (6) to $144.49(4)^{\circ}$, respectively. The $\mathrm{CHO}_{2}{ }^{-}$and $\mathrm{C}_{2} \mathrm{O}_{4}{ }^{2-}$ anions adopt $\mu_{3}$-bridging and $\mu_{4}$-chelating-bridging coordination modes, respectively, linking adjacent $\mathrm{Tb}^{\mathrm{III}}$ ions into a threedimensional 12-connected fcu topology with point symbol $\left(3^{24} \cdot 4^{36} \cdot 5^{6}\right)$. The title compound exhibits thermal stability up to $623 \mathrm{~K}$, and also displays strong green photoluminescence in the solid state at room temperature.

\section{Chemical context}

Owing to their high colour purity, high luminescence quantum yields, narrow bandwidths, relatively long lifetimes and large Stokes shifts arising from $4 f$ orbitals, coordination polymers of lanthanide(III) ions and organic linker ligands have received much attention from chemists during the past decade for the development of fluorescent probes and electroluminescent devices (Hasegawa \& Nakanishi, 2015). In particular, polymeric $\mathrm{Eu}^{\mathrm{III}}$ and $\mathrm{Tb}^{\mathrm{III}}$ compounds with a range of organic linker ligands are the most intense emitters among the lanthanide(III) series, and they have been developed extensively as ion sensing and optical materials (Cui et al., 2014). Lanthanide(III) ions are known to have a high affinity and preference for hard donor atoms. Thus, dicarboxylic acid ligands containing aliphatic, aromatic and $N$-heterocyclic moieties have been widely employed in the construction of luminescent lanthanide coordination polymers (So et al., 2015). Among the ligands in this class, for instance, terephthalic acid is known to provide an efficient energy transfer to support strong lanthanide(III)-centered luminescent emission via the 'antenna effect' (Samuel et al., 2009). On the other hand, small rigid planar species with versatile coordination oxygen donor sites such as oxalate, carbonate, nitrate, and formate anions are also a very important class of ligands for the preparation of 
lanthanide coordination polymers (Hong et al., 2014; Gupta et al., 2015). These small versatile ligands can bind to metals in different modes, resulting in the formation of multi-dimensional coordination networks with short intermetallic distances, which can aid the energy-transfer process between chromophoric antenna ligands and lanthanide(III) ions (Wang et al., 2012). In addition, the oxalate anion has proved to be an efficient sensitizer for lanthanide(III)-based emission (Cheng et al., 2007). Recently, many multi-dimensional luminescent lanthanide coordination polymers containing antenna and small rigid planar mixed ligands have been reported (Xu et al., 2013; Wang et al., 2013). However, only a few compounds with mixed small rigid planar ligands alone have been described in the literature (Zhang et al., 2007; Huang et al., 2013; Tang et al., 2014).

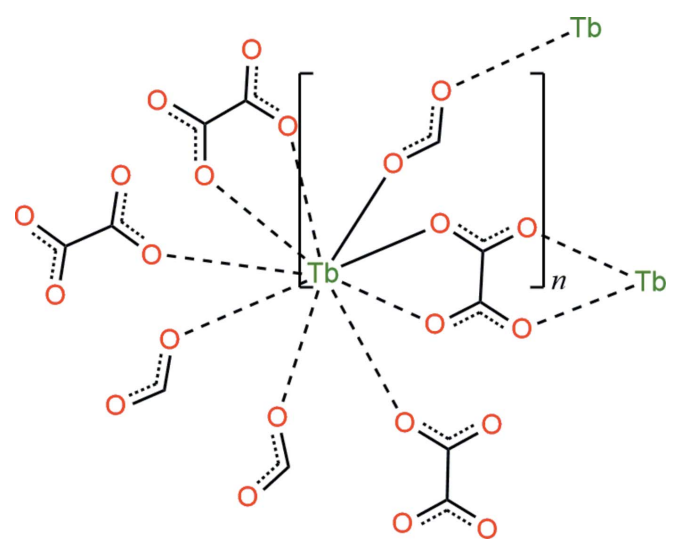

Herein, we report the synthesis and structure of a terbium(III) coordination polymer containing formate and oxalate mixed ligands, $\left[\mathrm{Tb}\left(\mathrm{CHO}_{2}\right)\left(\mathrm{C}_{2} \mathrm{O}_{4}\right)\right]_{n}$, (I), having a three-dimensional 12-connected fcu topology with point symbol $\left(3^{24} \cdot 4^{36} \cdot 5^{6}\right)$. The thermal stability and luminescent properties of compound (I) have also been investigated.

\section{Structural commentary}

Single crystal X-ray diffraction analysis revealed that (I) is isotypic in the orthorhombic Pnma space group with the $\mathrm{La}^{\mathrm{III}}$, $\mathrm{Ce}^{\mathrm{III}}$ and $\mathrm{Sm}^{\mathrm{III}}$ analogues (Romero et al., 1996). The asymmetric unit contains one $\mathrm{Tb}^{\mathrm{III}}$ ion, one formate anion, and half of an oxalate anion. As shown in Fig. 1, each $\mathrm{Tb}^{\mathrm{III}}$ ion is ninecoordinated in a distorted tricapped trigonal prismatic manner (Fig. 1) by two chelating carboxylate groups from two oxalate ligands, two carboxylate oxygen atoms from another two

Table 1

Selected bond lengths $(\AA)$.

\begin{tabular}{|c|c|c|c|}
\hline $\mathrm{Tb} 1-\mathrm{O} 1$ & $2.417(3)$ & $\mathrm{Tb} 1-\mathrm{O} 4^{\mathrm{iv}}$ & $2.4370(18)$ \\
\hline $\mathrm{Tb} 1-\mathrm{O} 1^{\mathrm{i}}$ & $2.478(3)$ & $\mathrm{Tb} 1-\mathrm{O} 4^{\mathrm{v}}$ & $2.4651(17)$ \\
\hline $\mathrm{Tb} 1-\mathrm{O} 2^{\mathrm{ii}}$ & $2.437(3)$ & $\mathrm{Tb} 1-\mathrm{O}^{\mathrm{vi}}$ & $2.4370(17)$ \\
\hline $\mathrm{Tb} 1-\mathrm{O} 3^{\mathrm{iii}}$ & $2.4165(19)$ & $\mathrm{Tb} 1-\mathrm{O} 4^{\mathrm{vii}}$ & $2.4651(17)$ \\
\hline $\mathrm{Tb} 1-\mathrm{O} 3$ & 2.4165 (19) & & \\
\hline
\end{tabular}

Symmetry codes: (i) $x-\frac{1}{2}, y,-z+\frac{3}{2}$; (ii) $x-\frac{1}{2}, y,-z+\frac{1}{2}$; (iii) $x,-y+\frac{3}{2}$, $z$; (iv) $-x,-y+1,-z+1 ; \quad$ (v) $\quad-x+\frac{1}{2},-y+1, z+\frac{1}{2} ; \quad$ (vi) $\quad-x, y+\frac{1}{2},-z+1$; (vii) $-x+\frac{1}{2}, y+\frac{1}{2}, z+\frac{1}{2}$.

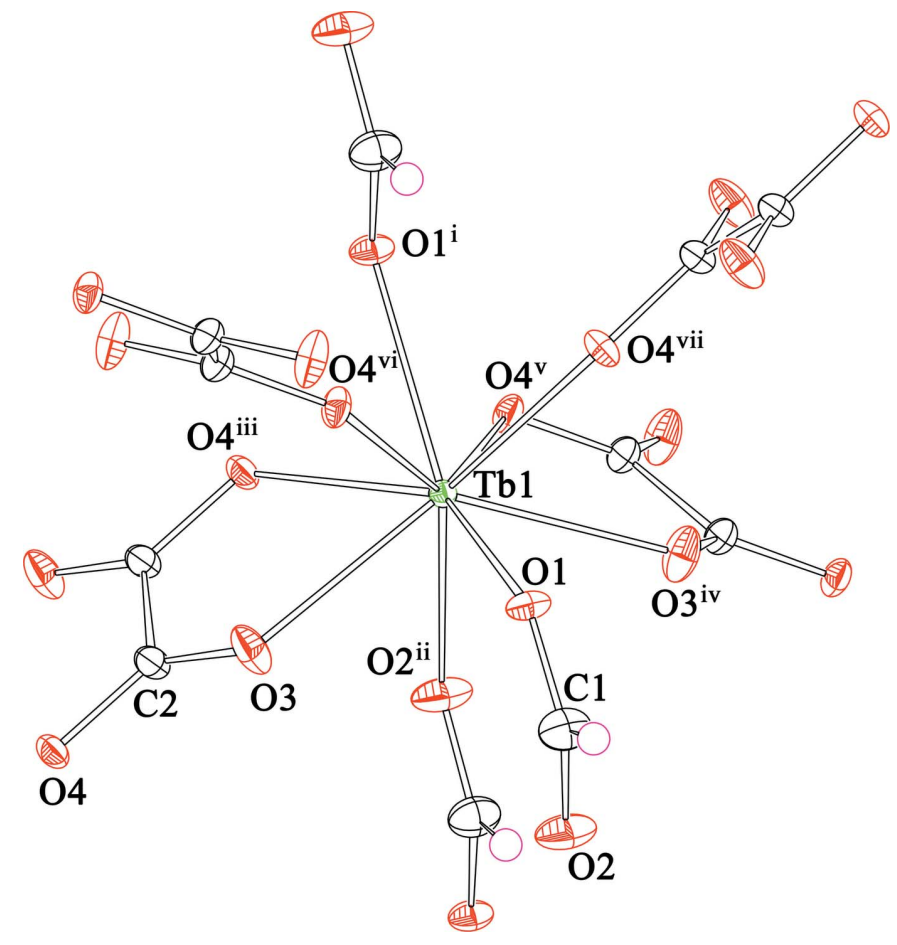

Figure 1

Coordination environment of the $\mathrm{Tb}^{\mathrm{III}}$ ion in (I). Displacement ellipsoids are drawn at the $50 \%$ probability level and $\mathrm{H}$ atoms are shown as small spheres of arbitrary radii. For symmetry codes, see Table 1.

oxalate ligands and three oxygen atoms from three formate ligands, with the $\mathrm{O}-\mathrm{Tb}-\mathrm{O}$ bond angles ranging from 64.53 (6) to $144.49(4)^{\circ}$. The $\mathrm{Tb}-\mathrm{O}$ bond lengths in (I) are in the range of 2.4165 (19) to 2.478 (3) $\AA$ (Table 1), which is in good agreement with the reported distances for other $\mathrm{Tb}^{\mathrm{III}}$

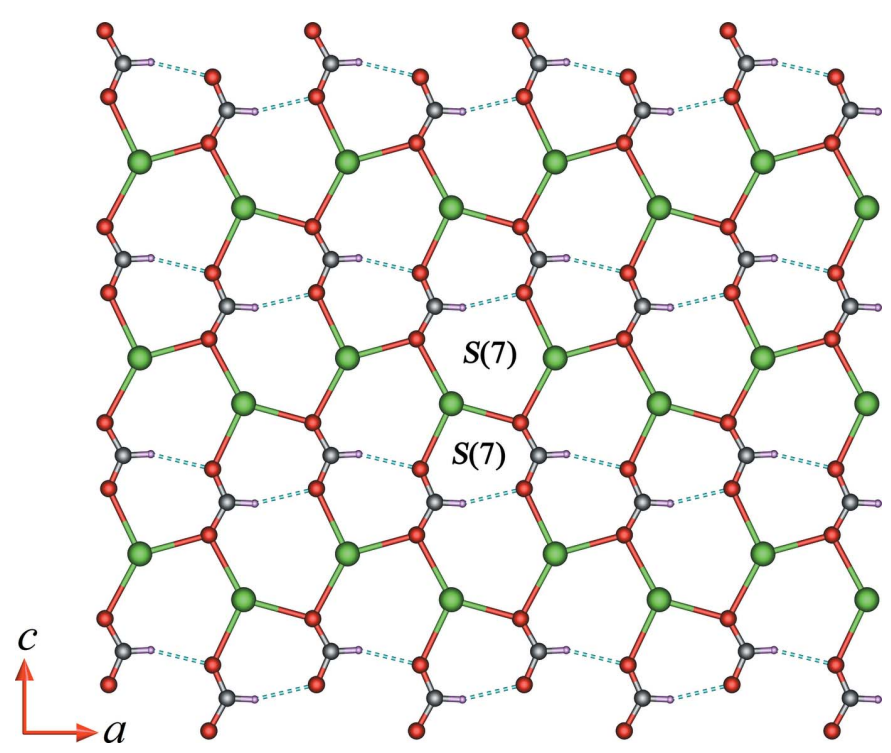

Figure 2

A view of the two-dimensional terbium-formate network in (I), showing the monolayer structure projected in the $a c$ plane. The dashed lines indicate the intralayer $\mathrm{C}-\mathrm{H} \cdots \mathrm{O}$ hydrogen bonds (Table 2). 

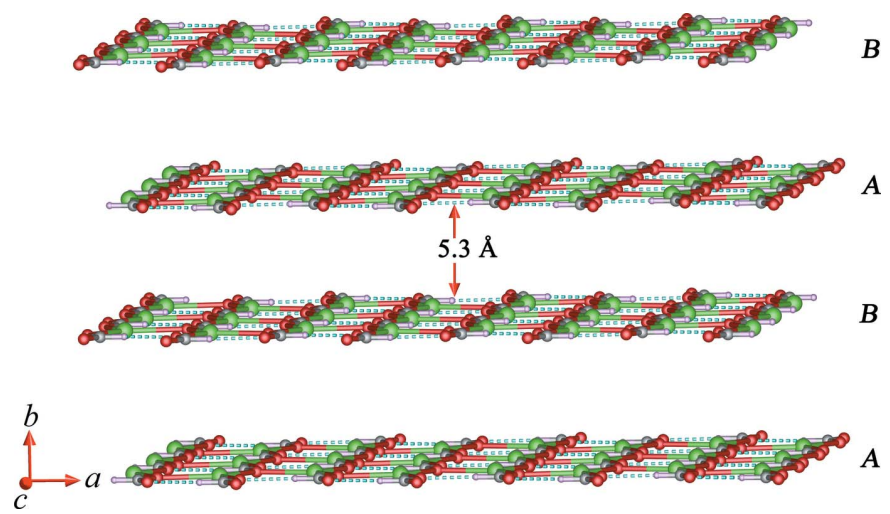

Figure 3

The terbium-formate layered structure viewed along the $c$ axis.

complexes containing oxygen donor ligands (Cheng et al., 2007; Zhu et al., 2007). All of the bond lengths and bond angles in the formate and oxalate anions are also within normal ranges (Rossin et al., 2012; Hong et al., 2014; Gupta et al., 2015). The coordination modes of the formate and oxalate ligands in (I) (Fig. 2) are commonly observed in lanthanide coordination polymers (Zhang et al., 2007; Rossin et al., 2012).

As shown in Fig. 2, each formate anion adopts a $\mu_{3}$-bridging coordination mode connecting three $\mathrm{Tb}^{\mathrm{III}}$ ions, forming a twodimensional (2-D) layer in the $a c$ plane. In the 2-D terbium-

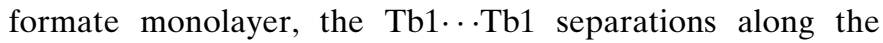
formate ligands in syn-anti and anti-anti O1,O2-bridging coordination modes (Rossin et al., 2012) are 6.1567 (3) and 6.6021 (2) $\AA$, respectively. The adjacent 2-D monolayers are stacked in an $-A B A$ - sequence running perpendicular to the $b$ axis with an interlayer spacing of $c a 5.3 \AA$ (Fig. 3). The oxalate ligand adopts a $\mu_{4}$-chelating-bridging coordination mode, linking four $\mathrm{Tb}^{\mathrm{III}}$ ions along the $a$ axis to form a threedimensional (3-D) terbium-oxalate open framework (Fig. 3). The Tb1 …Tb1 distance via the formate O1- and oxalate O4bridging ligands is 3.8309 (2) $\AA$ with the Tb1-O1-Tb1 and Tb1-O4-Tb1 bond angles being 103.00 (9) and $102.79(6)^{\circ}$, respectively. On the other hand, the channels in the 3-D open framework have an approximate rhombic shape with a

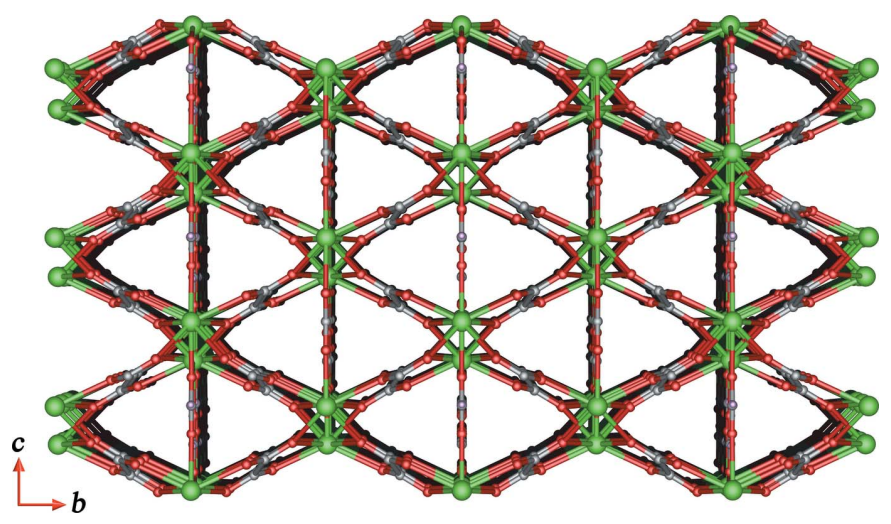

Figure 4

A perspective view along the $a$ axis of the three-dimensional framework.

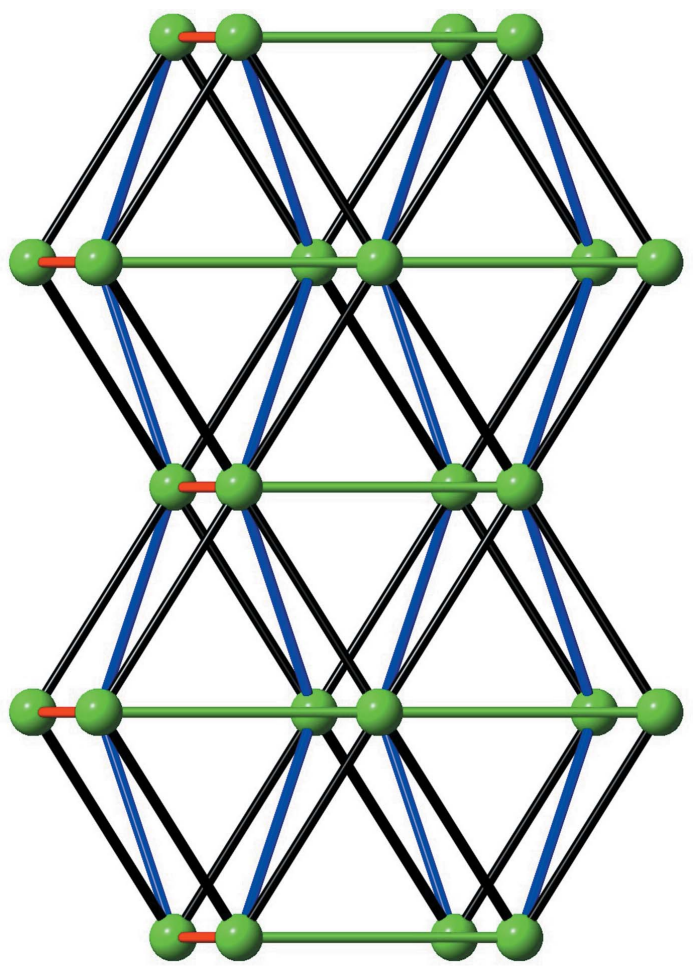

Figure 5

Schematic representation of the 12-connected fcu topology in (I).

Tb1…Tb1 separation of 6.2670 (2) $\AA$, and are cross-linked parallel to the $c$ axis by bridging formate ligands as shown in Fig. 4. The presence of guest molecules in the lattice as well as the formation of interpenetrated networks of (I) are thus prevented. Furthermore, the topology of the network in (I) was analysed using TOPOS (Blatov et al., 2000). As schematically depicted in Fig. 5, the overall framework can be defined as a 12 -connected fcu topology with point symbol $\left(3^{24} \cdot 4^{36} \cdot 5^{6}\right)$ by linking each adjacent layer of $\mathrm{Tb}^{\mathrm{III}}$ atoms via formate and oxalate ligands.

The infrared spectrum of (I) was collected from a polycrystalline sample pelletized with $\mathrm{KBr}$, in the range 4000$400 \mathrm{~cm}^{-1}$. This spectrum indicates the presence of the carboxylate groups of the ligands by appearance of the strong absorption bands at 1630 and $1315 \mathrm{~cm}^{-1}$ for the asymmetric $\left(v_{\text {asym }} \mathrm{COO}^{-}\right)$and the symmetric $\left(v_{\text {sym }} \mathrm{COO}^{-}\right)$carboxylate vibrations, respectively (Deacon \& Phillips, 1980). To examine the thermal stability of (I), thermogravimetric analysis was performed on a polycrystalline sample under a nitrogen atmosphere in the temperature range of 303-1273 K. There is no weight loss before $623 \mathrm{~K}$ due to the stability of the fcu-type 3-D frameworks. The decomposition of the framework, however, occurred rapidly at temperatures above $628 \mathrm{~K}$.

The photoluminescence properties of (I) were investigated in the solid state at room temperature. The emission spectrum is shown in Fig. 6. The emission spectrum upon excitation at $305 \mathrm{~nm}$ exhibits the characteristic $f-f$ transitions of $\mathrm{Tb}^{\mathrm{III}}$ ions (Bünzli, 2010). The emission peaks at 487, 543, 585, and $617 \mathrm{~nm}$ can be assigned to the ${ }^{5} D_{4} \rightarrow{ }^{7} F_{J}(J=6,5,4,3)$ transitions, respectively. The most intense transition is 
Table 2

Hydrogen-bond geometry $\left(\AA,^{\circ}\right)$.

\begin{tabular}{lllll}
\hline$D-\mathrm{H} \cdots A$ & $D-\mathrm{H}$ & $\mathrm{H} \cdots A$ & $D \cdots A$ & $D-\mathrm{H} \cdots A$ \\
\hline $\mathrm{C} 1-\mathrm{H} 1 \cdots \mathrm{O} 2^{\text {viii }}$ & 0.93 & 2.15 & $3.051(5)$ & 164 \\
\hline
\end{tabular}

Symmetry code: (viii) $x+\frac{1}{2},-y+\frac{3}{2},-z+\frac{1}{2}$.

${ }^{5} D_{4} \rightarrow{ }^{7} F_{5}$, which implies the emitted light is green. The emission lifetime of (I) is $1.79 \mathrm{~ms}$.

\section{Supramolecular features}

The two-dimensional terbium-formate monolayers are stabilized by weak intra-layer $\mathrm{C} 1-\mathrm{H} 1 \cdots \mathrm{O} 2{ }^{\text {viii }}$ hydrogen bonds giving $S(7)$ graph-set motifs (Bernstein et al., 1995), in which each formate anion acts as a donor and acceptor for one hydrogen bond (Table 2, Fig. 2).

\section{Database survey}

A search of the Cambridge Structural Database (Groom \& Allen, 2014) for lanthanide coordination polymers containing mixed oxalate and formate ligands gave four hits (RIFQIG, RIFRED, RIFRIH; Romero et al., 1996; RIFQIG01; Tan et al., 2009), which are isotypic with the title compound (I) as previously mentioned. The structures involving oxalate and acetate analogues have also been reported (AZOCIC; Di et al., 2011; Gutkowski et al., 2011; SOPPIX; Zhang et al., 2009; VORBUA; Koner \& Goldberg, 2009).

\section{Synthesis and crystallization}

All reagents were of analytical grade and were used as obtained from commercial sources without further purification. Synthesis of (I): $\mathrm{TbCl}_{3} \cdot 6 \mathrm{H}_{2} \mathrm{O}(0.187 \mathrm{~g}, 0.5 \mathrm{mmol})$, oxalic

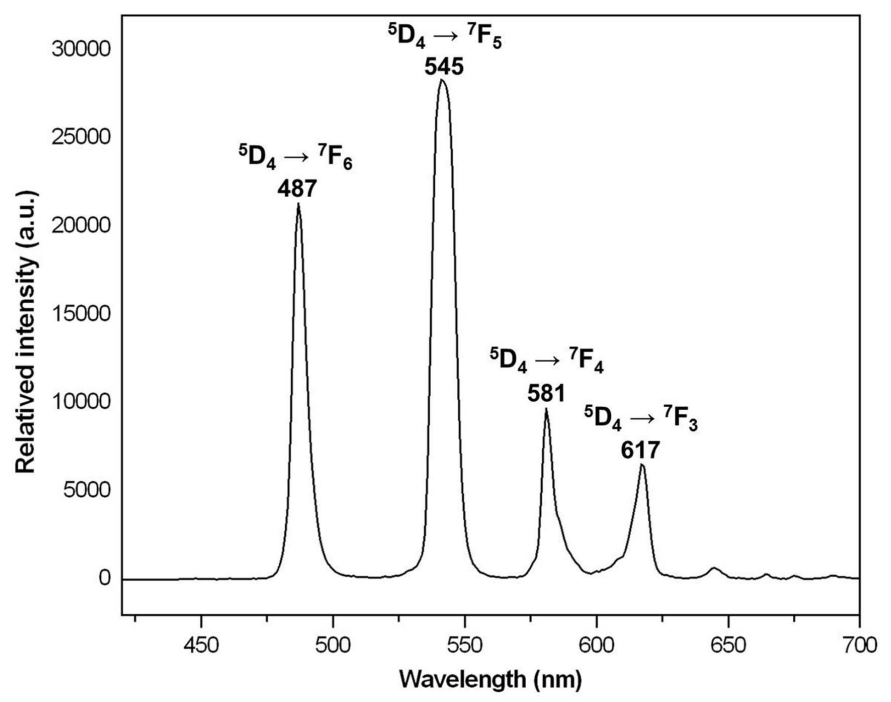

Figure 6

The solid-state emission spectrum of (I) at room temperature.
Table 3

Experimental details.

\begin{tabular}{|c|c|}
\hline \multicolumn{2}{|l|}{ Crystal data } \\
\hline Chemical formula & {$\left[\mathrm{Tb}\left(\mathrm{CHO}_{2}\right)\left(\mathrm{C}_{2} \mathrm{O}_{4}\right)\right]$} \\
\hline$M_{\mathrm{r}}$ & 291.96 \\
\hline Crystal system, space group & Orthorhombic, Pnma \\
\hline Temperature (K) & 296 \\
\hline$a, b, c(\AA)$ & $7.0138(3), 10.6077(4), 6.6021(2)$ \\
\hline$V\left(\AA^{3}\right)$ & $491.20(3)$ \\
\hline$Z$ & 4 \\
\hline Radiation type & Mo $K \alpha$ \\
\hline$\mu\left(\mathrm{mm}^{-1}\right)$ & 14.36 \\
\hline Crystal size $(\mathrm{mm})$ & $0.2 \times 0.12 \times 0.08$ \\
\hline \multicolumn{2}{|l|}{ Data collection } \\
\hline Diffractometer & Bruker D8 QUEST CMOS \\
\hline Absorption correction & $\begin{array}{l}\text { Multi-scan (SADABS; Bruker, } \\
\text { 2014) }\end{array}$ \\
\hline$T_{\min }, T_{\max }$ & $0.655,0.746$ \\
\hline $\begin{array}{l}\text { No. of measured, independent and } \\
\text { observed }[I>2 \sigma(I)] \text { reflections }\end{array}$ & $6517,638,594$ \\
\hline$R_{\text {int }}$ & 0.028 \\
\hline$(\sin \theta / \lambda)_{\max }\left(\AA^{-1}\right)$ & 0.666 \\
\hline \multicolumn{2}{|l|}{ Refinement } \\
\hline$R\left[F^{2}>2 \sigma\left(F^{2}\right)\right], w R\left(F^{2}\right), S$ & $0.012,0.025,1.10$ \\
\hline No. of reflections & 638 \\
\hline No. of parameters & 52 \\
\hline $\mathrm{H}$-atom treatment & $\mathrm{H}$-atom parameters constrained \\
\hline$\Delta \rho_{\max }, \Delta \rho_{\min }\left(\mathrm{e} \AA^{-3}\right)$ & $0.75,-0.63$ \\
\hline
\end{tabular}

Computer programs: APEX2 and SAINT (Bruker, 2014), SHELXS2014 (Sheldrick, 2008), SHELXL2014 (Sheldrick, 2015), OLEX2 (Dolomanov et al., 2009), publCIF (Westrip, 2010) and enCIFer (Allen et al., 2004).

acid (0.045 g, $0.5 \mathrm{mmol}), \mathrm{Na}_{2} \mathrm{CO}_{3}(0.011 \mathrm{~g}, 0.1 \mathrm{mmol})$, and a mixture $(1: 1 v / v)$ of $N, N^{\prime}$-dimethylformamide (DMF) and water $(6 \mathrm{ml})$ was sealed in a $23 \mathrm{ml}$ Teflon-lined stainless steel vessel and heated under autogenous pressure at $463 \mathrm{~K}$ for two days. After the reactor was cooled to room temperature, colorless block-shaped crystals were filtered off and dried in air. Yield: $0.118 \mathrm{~g}$ (63\% based on the $\mathrm{Tb}^{\mathrm{III}}$ source). Analysis (\%) calculated for $\mathrm{C}_{3} \mathrm{HO}_{6} \mathrm{~Tb}$ (291.96): C, 12.34; $\mathrm{H}, 0.35 \%$. Found: C, 12.40; H, 0.33\%. IR (KBr, cm $\left.{ }^{-1}\right): 2823(w), 2491(w)$, $1630(s), 1440(w), 1315(s), 1022(m), 914(w), 795(s), 611(w)$, $492(s), 408(w)$.

\section{Refinement}

Crystal data, data collection and structure refinement details are summarized in Table 3 . The formate $\mathrm{H}$ atom was found in a difference electron-density map and was refined using a riding-model approximation, with $\mathrm{C}-\mathrm{H}=0.93 \AA$ and with $U_{\text {iso }}(\mathrm{H})=1.2 U_{\mathrm{eq}}(\mathrm{C})$.

\section{Acknowledgements}

This research was supported financially by the National Research Council of Thailand through the Thammasat University Research Scholar (No. 216919). We thank Central Scientific Instrument Center (CSIC), Faculty of Science and Technology, Thammasat University, for providing access to the equipment. 


\section{References}

Allen, F. H., Johnson, O., Shields, G. P., Smith, B. R. \& Towler, M. (2004). J. Appl. Cryst. 37, 335-338.

Bernstein, J., Davis, R. E., Shimoni, L. \& Chang, N.-L. (1995). Angew. Chem. Int. Ed. Engl. 34, 1555-1573.

Blatov, V. A., Shevchenko, A. P. \& Serezhkin, V. N. (2000). J. Appl. Cryst. 33, 1193.

Bruker (2014). APEX2, SADABS and SAINT. Bruker AXS Inc., Madison, Wisconsin, USA.

Bünzli, J. G. (2010). Chem. Rev. 110, 2729-2755.

Cheng, J. W., Zheng, S. T. \& Yang, G. Y. (2007). Dalton Trans. pp. 4059-4066.

Cui, Y., Chen, B. \& Qian, G. (2014). Coord. Chem. Rev. 273-274, 7686.

Deacon, G. B. \& Phillips, R. J. (1980). Coord. Chem. Rev. 33, $227-$ 250.

Di, W.-J., Lan, S.-M., Zhang, Q. \& Liang, Y.-X. (2011). Acta Cryst. E67, m1436-m1437.

Dolomanov, O. V., Bourhis, L. J., Gildea, R. J., Howard, J. A. K. \& Puschmann, H. (2009). J. Appl. Cryst. 42, 339-341.

Groom, C. R. \& Allen, F. H. (2014). Angew. Chem. Int. Ed. 53, 662671.

Gupta, S. K., Rajeshwari, B., Achary, S. N., Patwe, S. J., Tyagi, A. K., Natarajan, V. \& Kadam, R. M. (2015). Eur. J. Inorg. Chem. pp. 4429-4436.

Gutkowski, K., Freire, E. \& Baggio, R. (2011). Acta Cryst. C67, m77m80.

Hasegawa, Y. \& Nakanishi, T. (2015). RSC Adv. 5, 338-353.

Hong, F., Zhou, L., Li, L., Xia, Q. \& Ye, M. (2014). Mater. Res. Bull. 60, 252-257.
Huang, D.-Q., Zhang, H., Sheng, L.-Q., Liu, Z.-D., Xu, H.-J. \& Fan, S.-H. (2013). Chin. J. Inorg. Chem. 29, 1-6.

Koner, R. \& Goldberg, I. (2009). Acta Cryst. C65, m160-m164.

Romero, S., Mosset, A. \& Trombe, J. C. (1996). J. Solid State Chem. 127, 256-266.

Rossin, A., Giambastiani, G., Peruzzini, M. \& Sessoli, R. (2012). Inorg. Chem. 51, 6962-6968.

Samuel, A. P. S., Xu, J. \& Raymond, K. N. (2009). Inorg. Chem. 48 , 687-698.

Sheldrick, G. M. (2008). Acta Cryst. A64, 112-122.

Sheldrick, G. M. (2015). Acta Cryst. C71, 3-8.

So, M. C., Wiederrecht, G. P., Mondloch, J. E., Hupp, J. T. \& Farha, O. K. (2015). Chem. Commun. 51, 3501-3510.

Tan, J. C., Furman, J. D. \& Cheetham, A. K. (2009). J. Am. Chem. Soc. 131, 14252-14254.

Tang, Q., Liu, Y., Liu, S., He, D., Miao, J., Wang, X., Yang, G., Shi, Z. \& Zheng, Z. (2014). J. Am. Chem. Soc. 136, 12444-12449.

Wang, P., Fan, R.-Q., Liu, X.-R., Wang, L.-Y., Yang, Y.-L., Cao, W.-W., Yang, B., Hasi, W., Su, Q. \& Mu, Y. (2013). CrystEngComm, 15, 1931-1949.

Wang, H., Liu, S.-J., Tian, D., Jia, J.-M. \& Hu, T.-L. (2012). Cryst. Growth Des. 12, 3263-3270.

Westrip, S. P. (2010). J. Appl. Cryst. 43, 920-925.

Xu, X., Zhou, Y., Huang, D., Xiong, W., Su, M., Wang, K., Han, S. \& Hong, M. (2013). Cryst. Growth Des. 13, 5420-5432.

Zhang, L.-Z., Gu, W., Li, B., Liu, X. \& Liao, D.-Z. (2007). Inorg. Chem. 46, 622-624.

Zhang, X., Xing, Y., Wang, C., Han, J., Li, J., Ge, M., Zeng, X. \& Niu, S. (2009). Inorg. Chim. Acta, 362, 1058-1064.

Zhu, W. H., Wang, Z. M. \& Gao, S. (2007). Inorg. Chem. 46, 13371342. 


\section{supporting information}

Acta Cryst. (2016). E72, 87-91 [doi:10.1107/S205698901502397X]

Crystal structure of a mixed-ligand terbium(III) coordination polymer containing oxalate and formate ligands, having a three-dimensional fcu topology

\section{Chainok Kittipong, Phailyn Khemthong, Filip Kielar and Yan Zhou}

Computing details

Data collection: APEX2 (Bruker, 2014); cell refinement: SAINT (Bruker, 2014); data reduction: SAINT (Bruker, 2014); program(s) used to solve structure: SHELXS2014 (Sheldrick, 2008); program(s) used to refine structure: SHELXL2014 (Sheldrick, 2015); molecular graphics: OLEX2 (Dolomanov et al., 2009); software used to prepare material for publication: publCIF (Westrip, 2010) and enCIFer (Allen et al., 2004).

Poly $\left[\left(\mu_{3}\right.\right.$-formato $)\left(\mu_{4}\right.$-oxalato $)$ terbium(III)]

Crystal data

$\left[\mathrm{Tb}\left(\mathrm{CHO}_{2}\right)\left(\mathrm{C}_{2} \mathrm{O}_{4}\right)\right]$

$M_{r}=291.96$

Orthorhombic, Pnma

$a=7.0138(3) \AA$

$b=10.6077$ (4) $\AA$

$c=6.6021(2) \AA$

$V=491.20(3) \AA^{3}$

$Z=4$

$F(000)=528$

\section{Data collection}

Bruker D8 QUEST CMOS diffractometer

Radiation source: microfocus sealed x-ray tube, Incoatec I $\mu$ us

Graphite Double Bounce Multilayer Mirror monochromator

Detector resolution: 10.5 pixels $\mathrm{mm}^{-1}$

$\omega$ and $\varphi$ scans

Absorption correction: multi-scan

(SADABS; Bruker, 2014)

Refinement

Refinement on $F^{2}$

Least-squares matrix: full

$R\left[F^{2}>2 \sigma\left(F^{2}\right)\right]=0.012$

$w R\left(F^{2}\right)=0.025$

$S=1.10$

638 reflections

52 parameters
$D_{\mathrm{x}}=3.948 \mathrm{Mg} \mathrm{m}^{-3}$

Mo $K \alpha$ radiation, $\lambda=0.71073 \AA$

Cell parameters from 3952 reflections

$\theta=3.6-28.3^{\circ}$

$\mu=14.36 \mathrm{~mm}^{-1}$

$T=296 \mathrm{~K}$

Block, colourless

$0.2 \times 0.12 \times 0.08 \mathrm{~mm}$

$T_{\min }=0.655, T_{\max }=0.746$

6517 measured reflections

638 independent reflections

594 reflections with $I>2 \sigma(I)$

$R_{\text {int }}=0.028$

$\theta_{\max }=28.3^{\circ}, \theta_{\min }=3.6^{\circ}$

$h=-9 \rightarrow 9$

$k=-13 \rightarrow 14$

$l=-8 \rightarrow 8$

0 restraints

Primary atom site location: structure-invariant direct methods

Secondary atom site location: difference Fourier map

Hydrogen site location: inferred from neighbouring sites 
H-atom parameters constrained

$w=1 /\left[\sigma^{2}\left(F_{\mathrm{o}}^{2}\right)+(0.0092 P)^{2}+0.8666 P\right]$

where $P=\left(F_{\mathrm{o}}^{2}+2 F_{\mathrm{c}}^{2}\right) / 3$

$$
\begin{aligned}
& (\Delta / \sigma)_{\max }=0.001 \\
& \Delta \rho_{\max }=0.75 \mathrm{e} \AA^{-3} \\
& \Delta \rho_{\min }=-0.63 \mathrm{e} \AA^{-3}
\end{aligned}
$$

\section{Special details}

Experimental. SADABS-2014 (Bruker, 2014) was used for absorption correction. wR2(int) was 0.0566 before and 0.0416 after correction. The ratio of minimum to maximum transmission is 0.8789 . The $\lambda / 2$ correction factor is 0.00150 . Geometry. All esds (except the esd in the dihedral angle between two 1.s. planes) are estimated using the full covariance matrix. The cell esds are taken into account individually in the estimation of esds in distances, angles and torsion angles; correlations between esds in cell parameters are only used when they are defined by crystal symmetry. An approximate (isotropic) treatment of cell esds is used for estimating esds involving l.s. planes.

Refinement. Refinement of $\mathrm{F}^{2}$ against ALL reflections. The weighted R-factor $\mathrm{wR}$ and goodness of fit $\mathrm{S}$ are based on $\mathrm{F}^{2}$, conventional R-factors $R$ are based on $F$, with $F$ set to zero for negative $F^{2}$. The threshold expression of $F^{2}>2 s i g m a\left(F^{2}\right)$ is used only for calculating R-factors ( $\mathrm{gt}$ ) etc. and is not relevant to the choice of reflections for refinement. R-factors based on $\mathrm{F}^{2}$ are statistically about twice as large as those based on F, and R-factors based on ALL data will be even larger.

Fractional atomic coordinates and isotropic or equivalent isotropic displacement parameters $\left(\AA^{2}\right)$

\begin{tabular}{lllll}
\hline & $x$ & $y$ & $z$ & $U_{\text {iso }} * / U_{\text {eq }}$ \\
\hline Tb1 & $0.20226(2)$ & 0.7500 & $0.63323(2)$ & $0.00749(6)$ \\
O1 & $0.5347(4)$ & 0.7500 & $0.5364(4)$ & $0.0132(5)$ \\
O2 & $0.5527(4)$ & 0.7500 & $0.2000(4)$ & $0.0237(7)$ \\
O3 & $0.2384(3)$ & $0.54490(18)$ & $0.4786(3)$ & $0.0186(4)$ \\
O4 & $0.0873(3)$ & $0.37671(16)$ & $0.3522(3)$ & $0.0120(4)$ \\
$\mathrm{C} 1$ & $0.6227(6)$ & 0.7500 & $0.3693(6)$ & $0.0197(8)$ \\
$\mathrm{H} 1$ & 0.7551 & 0.7500 & 0.3761 & $0.024 *$ \\
$\mathrm{C} 2$ & $0.0956(4)$ & $0.4788(2)$ & $0.4518(4)$ & $0.0124(5)$ \\
\hline
\end{tabular}

Atomic displacement parameters $\left(\AA^{2}\right)$

\begin{tabular}{lllllll}
\hline & $U^{11}$ & $U^{22}$ & $U^{33}$ & $U^{12}$ & $U^{13}$ & $U^{23}$ \\
\hline $\mathrm{Tb} 1$ & $0.00799(8)$ & $0.00750(8)$ & $0.00698(9)$ & 0.000 & $-0.00049(7)$ & 0.000 \\
$\mathrm{O} 1$ & $0.0110(13)$ & $0.0209(14)$ & $0.0076(13)$ & 0.000 & $0.0013(10)$ & 0.000 \\
$\mathrm{O} 2$ & $0.0235(16)$ & $0.0381(18)$ & $0.0096(14)$ & 0.000 & $-0.0010(12)$ & 0.000 \\
$\mathrm{O} 3$ & $0.0133(9)$ & $0.0143(9)$ & $0.0282(11)$ & $-0.0028(7)$ & $0.0010(8)$ & $-0.0093(9)$ \\
$\mathrm{O} 4$ & $0.0136(8)$ & $0.0093(8)$ & $0.0131(9)$ & $-0.0011(7)$ & $0.0027(7)$ & $-0.0041(7)$ \\
$\mathrm{C} 1$ & $0.0141(17)$ & $0.029(2)$ & $0.016(2)$ & 0.000 & $-0.0009(16)$ & 0.000 \\
$\mathrm{C} 2$ & $0.0151(12)$ & $0.0112(11)$ & $0.0109(12)$ & $0.0003(10)$ & $-0.0002(10)$ & $-0.0020(10)$ \\
\hline
\end{tabular}

Geometric parameters $\left(\AA,{ }^{\circ}\right)$

\begin{tabular}{llll}
\hline $\mathrm{Tb} 1-\mathrm{O} 1$ & $2.417(3)$ & $\mathrm{Tb} 1-\mathrm{O} 4^{\mathrm{vii}}$ & $2.4651(17)$ \\
$\mathrm{Tb} 1-\mathrm{O} 1^{\mathrm{i}}$ & $2.478(3)$ & $\mathrm{O} 1-\mathrm{C} 1$ & $1.265(5)$ \\
$\mathrm{Tb} 1-\mathrm{O} 2^{\mathrm{ii}}$ & $2.437(3)$ & $\mathrm{O} 2-\mathrm{C} 1$ & $1.221(5)$ \\
$\mathrm{Tb} 1-\mathrm{O} 3^{\mathrm{iii}}$ & $2.4165(19)$ & $\mathrm{O} 3-\mathrm{C} 2$ & $1.235(3)$ \\
$\mathrm{Tb} 1-\mathrm{O} 3$ & $2.4165(19)$ & $\mathrm{O} 4-\mathrm{C} 2$ & $1.268(3)$ \\
$\mathrm{Tb} 1-\mathrm{O} 4^{\mathrm{iv}}$ & $2.4370(18)$ & $\mathrm{C} 1-\mathrm{H} 1$ & 0.9300 \\
$\mathrm{~Tb} 1-\mathrm{O} 4^{\mathrm{v}}$ & $2.4651(17)$ & $\mathrm{C} 2-\mathrm{C} 2^{\mathrm{iv}}$ & $1.551(5)$ \\
$\mathrm{Tb} 1-\mathrm{O} 4^{\mathrm{vi}}$ & $2.4370(17)$ & &
\end{tabular}




\begin{tabular}{|c|c|c|c|}
\hline 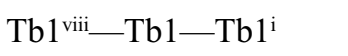 & $132.533(9)$ & $\mathrm{O} 4^{\mathrm{vi}}-\mathrm{Tb} 1-\mathrm{Tb} 1^{\mathrm{viii}}$ & $138.32(4)$ \\
\hline $\mathrm{O} 1-\mathrm{Tb} 1-\mathrm{Tb} 1^{\mathrm{viii}}$ & $39.06(6)$ & $\mathrm{O} 4^{\mathrm{v}}-\mathrm{Tb} 1-\mathrm{Tb} 1^{\mathrm{viii}}$ & $38.34(4)$ \\
\hline 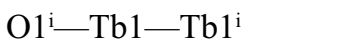 & $37.94(6)$ & 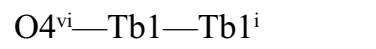 & $38.87(4)$ \\
\hline $\mathrm{O} 1-\mathrm{Tb} 1-\mathrm{Tb} 1^{\mathrm{i}}$ & $171.60(6)$ & $\mathrm{O} 4^{\mathrm{vii}}-\mathrm{Tb} 1-\mathrm{Tb} 1^{\mathrm{i}}$ & $108.19(4)$ \\
\hline 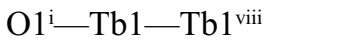 & $94.59(6)$ & $\mathrm{O} 4^{\mathrm{iv}}-\mathrm{Tb} 1-\mathrm{Tb} 1^{\mathrm{i}}$ & $38.87(4)$ \\
\hline $\mathrm{O} 1-\mathrm{Tb} 1-\mathrm{O} 1^{\mathrm{i}}$ & $133.65(7)$ & $\mathrm{O} 4^{\mathrm{iv}}-\mathrm{Tb} 1-\mathrm{Tb} 1^{\mathrm{viii}}$ & $138.33(4)$ \\
\hline $\mathrm{O} 1-\mathrm{Tb} 1-\mathrm{O} 2^{\mathrm{ii}}$ & $100.16(9)$ & $\mathrm{O} 4^{\mathrm{v}}-\mathrm{Tb} 1-\mathrm{Tb} 1^{\mathrm{i}}$ & $108.19(4)$ \\
\hline $\mathrm{O} 1-\mathrm{Tb} 1-\mathrm{O} 4^{\mathrm{vii}}$ & $65.01(6)$ & $\mathrm{O} 4^{\mathrm{vii}}-\mathrm{Tb} 1-\mathrm{Tb} 1^{\mathrm{viii}}$ & $38.34(4)$ \\
\hline $\mathrm{O} 1-\mathrm{Tb} 1-\mathrm{O} 4^{\mathrm{vi}}$ & $144.49(4)$ & $\mathrm{O} 4^{\mathrm{iv}}-\mathrm{Tb} 1-\mathrm{O} 1^{\mathrm{i}}$ & $64.53(6)$ \\
\hline $\mathrm{O} 1-\mathrm{Tb} 1-\mathrm{O} 4^{\mathrm{v}}$ & $65.01(6)$ & 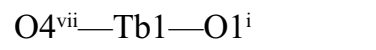 & $76.57(6)$ \\
\hline $\mathrm{O} 1-\mathrm{Tb} 1-\mathrm{O} 4^{\mathrm{iv}}$ & $144.49(4)$ & 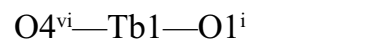 & $64.53(6)$ \\
\hline 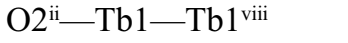 & $139.22(7)$ & 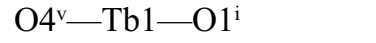 & $76.57(6)$ \\
\hline $\mathrm{O} 2^{\mathrm{ii}-}-\mathrm{Tb} 1-\mathrm{Tb} 1^{\mathrm{i}}$ & $88.25(7)$ & $\mathrm{O} 4^{\mathrm{vi}-\mathrm{Tb}} 1-\mathrm{O} 2^{\mathrm{ii}}$ & $71.16(7)$ \\
\hline 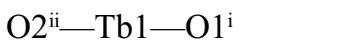 & $126.19(9)$ & $\mathrm{O} 4^{\mathrm{iv}}-\mathrm{Tb} 1-\mathrm{O} 2^{\mathrm{ii}}$ & $71.16(7)$ \\
\hline $\mathrm{O} 2^{\mathrm{ii}-}-\mathrm{Tb} 1-\mathrm{O} 4^{\mathrm{v}}$ & $141.92(5)$ & $\mathrm{O} 4^{\mathrm{v}}-\mathrm{Tb} 1-\mathrm{O} 4^{\mathrm{vii}}$ & $66.08(8)$ \\
\hline $\mathrm{O} 2^{\mathrm{ii}-}-\mathrm{Tb} 1-\mathrm{O} 4^{\mathrm{vii}}$ & $141.92(5)$ & $\mathrm{O} 4^{\mathrm{vi}-\mathrm{Tb}} 1-\mathrm{O} 4^{\mathrm{v}}$ & $140.95(3)$ \\
\hline $\mathrm{O} 3-\mathrm{Tb} 1-\mathrm{Tb} 1^{\mathrm{viii}}$ & $94.25(5)$ & $\mathrm{O} 4^{\mathrm{iv}}-\mathrm{Tb} 1-\mathrm{O} 4^{\mathrm{vi}}$ & $66.94(8)$ \\
\hline 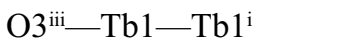 & $105.42(5)$ & $\mathrm{O} 4^{\mathrm{iv}}-\mathrm{Tb} 1-\mathrm{O} 4^{\mathrm{v}}$ & $100.09(6)$ \\
\hline $\mathrm{O} 3^{\mathrm{iii}}-\mathrm{Tb} 1-\mathrm{Tb} 1^{\mathrm{viii}}$ & $94.25(5)$ & 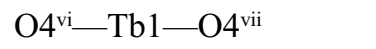 & $100.09(6)$ \\
\hline $\mathrm{O} 3-\mathrm{Tb} 1-\mathrm{Tb} 1^{\mathrm{i}}$ & $105.42(5)$ & $\mathrm{O} 4^{\mathrm{iv}}-\mathrm{Tb} 1-\mathrm{O} 4^{\mathrm{vii}}$ & $140.95(3)$ \\
\hline $\mathrm{O} 3-\mathrm{Tb} 1-\mathrm{O} 1$ & $77.72(5)$ & $\mathrm{Tb} 1-\mathrm{O} 1-\mathrm{Tb} 1^{\text {viii }}$ & $103.00(9)$ \\
\hline $\mathrm{O} 3-\mathrm{Tb} 1-\mathrm{O} 1^{\mathrm{i}}$ & $114.93(5)$ & $\mathrm{C} 1-\mathrm{O} 1-\mathrm{Tb} 1^{\mathrm{viii}}$ & $122.4(2)$ \\
\hline 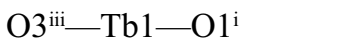 & $114.93(5)$ & $\mathrm{C} 1-\mathrm{O} 1-\mathrm{Tb} 1$ & $134.6(2)$ \\
\hline $\mathrm{O}^{3 i i i}-\mathrm{Tb} 1-\mathrm{O} 1$ & $77.72(5)$ & $\mathrm{C} 1-\mathrm{O} 2-\mathrm{Tb} 1^{\mathrm{ix}}$ & $130.8(3)$ \\
\hline $\mathrm{O} 3-\mathrm{Tb} 1-\mathrm{O} 2^{\mathrm{ii}}$ & $70.35(5)$ & $\mathrm{C} 2-\mathrm{O} 3-\mathrm{Tb} 1$ & $119.13(17)$ \\
\hline 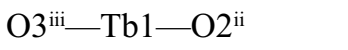 & $70.35(5)$ & $\mathrm{Tb} 1^{\mathrm{iv}}-\mathrm{O} 4-\mathrm{Tb} 1^{\mathrm{x}}$ & $102.79(6)$ \\
\hline $\mathrm{O} 3-\mathrm{Tb} 1-\mathrm{O} 3^{\mathrm{iii}}$ & $128.40(10)$ & $\mathrm{C} 2-\mathrm{O} 4-\mathrm{Tb} 1^{\mathrm{x}}$ & $137.90(16)$ \\
\hline $\mathrm{O} 3-\mathrm{Tb} 1-\mathrm{O} 4^{\mathrm{vii}}$ & $132.53(6)$ & $\mathrm{C} 2-\mathrm{O} 4-\mathrm{Tb} 1^{\mathrm{iv}}$ & $119.27(16)$ \\
\hline $\mathrm{O} 3-\mathrm{Tb} 1-\mathrm{O} 4^{\mathrm{vi}}$ & $126.90(6)$ & $\mathrm{O} 1-\mathrm{C} 1-\mathrm{H} 1$ & 116.5 \\
\hline $\mathrm{O} 3^{\mathrm{iii}}-\mathrm{Tb} 1-\mathrm{O} 4^{\mathrm{iv}}$ & $126.90(6)$ & $\mathrm{O} 2-\mathrm{C} 1-\mathrm{O} 1$ & $127.1(4)$ \\
\hline $\mathrm{O} 3^{\mathrm{iii}}-\mathrm{Tb} 1-\mathrm{O} 4^{\mathrm{vi}}$ & $66.88(6)$ & $\mathrm{O} 2-\mathrm{C} 1-\mathrm{H} 1$ & 116.5 \\
\hline 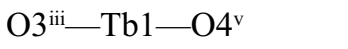 & $132.52(6)$ & $\mathrm{O} 3-\mathrm{C} 2-\mathrm{O} 4$ & $126.6(2)$ \\
\hline 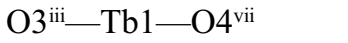 & $72.19(6)$ & $\mathrm{O} 3-\mathrm{C} 2-\mathrm{C} 2^{\mathrm{iv}}$ & $118.5(3)$ \\
\hline $\mathrm{O} 3-\mathrm{Tb} 1-\mathrm{O} 4^{\mathrm{v}}$ & $72.19(6)$ & $\mathrm{O} 4-\mathrm{C} 2-\mathrm{C}^{\mathrm{iv}}$ & $114.9(3)$ \\
\hline $\mathrm{O} 3-\mathrm{Tb} 1-\mathrm{O} 4^{\mathrm{iv}}$ & $66.88(6)$ & & \\
\hline $\mathrm{Tb} 1 \mathrm{viii}-\mathrm{Tb} 1-\mathrm{O} 1-\mathrm{C} 1$ & 180.0 & 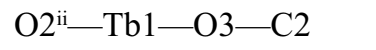 & $-67.5(2)$ \\
\hline 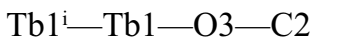 & $14.9(2)$ & $\mathrm{O} 3^{\mathrm{iii}}-\mathrm{Tb} 1-\mathrm{O} 1-\mathrm{Tb} 1^{\mathrm{viii}}$ & $112.87(5)$ \\
\hline $\mathrm{Tb} 1^{\mathrm{viii}}-\mathrm{Tb} 1-\mathrm{O} 3-\mathrm{C} 2$ & $151.3(2)$ & $\mathrm{O} 3-\mathrm{Tb} 1-\mathrm{O} 1-\mathrm{Tb} 1^{\mathrm{viii}}$ & $-112.87(5)$ \\
\hline $\mathrm{Tb} 1-\mathrm{O} 1-\mathrm{C} 1-\mathrm{O} 2$ & 0.0 & 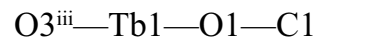 & $-67.13(5)$ \\
\hline $\mathrm{Tb} 1^{\mathrm{vii}}-\mathrm{O} 1-\mathrm{C} 1-\mathrm{O} 2$ & 180.0 & $\mathrm{O} 3-\mathrm{Tb} 1-\mathrm{O} 1-\mathrm{C} 1$ & $67.13(5)$ \\
\hline $\mathrm{Tb} 1^{\mathrm{ix}}-\mathrm{O} 2-\mathrm{C} 1-\mathrm{O} 1$ & 180.0 & $\mathrm{O} 3^{\mathrm{iii}}-\mathrm{Tb} 1-\mathrm{O} 3-\mathrm{C} 2$ & $-109.9(2)$ \\
\hline $\mathrm{Tb} 1-\mathrm{O} 3-\mathrm{C} 2-\mathrm{O} 4$ & $171.1(2)$ & $\mathrm{O} 4^{\mathrm{v}}-\mathrm{Tb} 1-\mathrm{O} 1-\mathrm{Tb} 1^{\mathrm{viii}}$ & $-36.98(5)$ \\
\hline $\mathrm{Tb} 1-\mathrm{O} 3-\mathrm{C} 2-\mathrm{C} 2^{\mathrm{iv}}$ & $-9.4(4)$ & 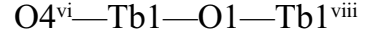 & $108.29(11)$ \\
\hline $\mathrm{Tb} 1^{\mathrm{x}}-\mathrm{O} 4-\mathrm{C} 2-\mathrm{O} 3$ & $-6.7(5)$ & $\mathrm{O} 4^{\mathrm{vii}}-\mathrm{Tb} 1-\mathrm{O} 1-\mathrm{Tb} 1^{\mathrm{viii}}$ & $36.98(5)$ \\
\hline $\mathrm{Tb}^{\mathrm{iv}}-\mathrm{O} 4-\mathrm{C} 2-\mathrm{O} 3$ & $170.9(2)$ & $\mathrm{O} 4^{\mathrm{iv}}-\mathrm{Tb} 1-\mathrm{O} 1-\mathrm{Tb} 1^{\mathrm{viii}}$ & $-108.29(11)$ \\
\hline $\mathrm{Tb} 1^{\mathrm{iv}}-\mathrm{O} 4-\mathrm{C} 2-\mathrm{C} 2^{\mathrm{iv}}$ & $-8.7(4)$ & $\mathrm{O} 4^{\mathrm{v}}-\mathrm{Tb} 1-\mathrm{O} 1-\mathrm{C} 1$ & $143.02(5)$ \\
\hline $\mathrm{Tb}^{\mathrm{x}}-\mathrm{O} 4-\mathrm{C} 2-\mathrm{C} 2^{\mathrm{iv}}$ & $173.74(18)$ & $\mathrm{O} 4{ }^{\mathrm{vi}}-\mathrm{Tb} 1-\mathrm{O} 1-\mathrm{C} 1$ & $-71.71(11)$ \\
\hline
\end{tabular}




\begin{tabular}{|c|c|c|c|}
\hline $\mathrm{O} 1^{\mathrm{i}}-\mathrm{Tb} 1-\mathrm{O} 1-\mathrm{Tb} 1^{\mathrm{viii}}$ & 0.0 & $\mathrm{O} 4^{\mathrm{vii}}-\mathrm{Tb} 1-\mathrm{O} 1-\mathrm{C} 1$ & $-143.02(5)$ \\
\hline $\mathrm{O} 1 \mathrm{i}-\mathrm{Tb} 1-\mathrm{O} 1-\mathrm{C} 1$ & 180.0 & $\mathrm{O} 4^{\mathrm{iv}}-\mathrm{Tb} 1-\mathrm{O} 1-\mathrm{C} 1$ & $71.71(11)$ \\
\hline $\mathrm{O} 11^{\mathrm{i}}-\mathrm{Tb} 1-\mathrm{O} 3-\mathrm{C} 2$ & $54.2(2)$ & $\mathrm{O} 4^{\mathrm{iv}}-\mathrm{Tb} 1-\mathrm{O} 3-\mathrm{C} 2$ & $9.75(19)$ \\
\hline $\mathrm{O} 1-\mathrm{Tb} 1-\mathrm{O} 3-\mathrm{C} 2$ & $-173.1(2)$ & 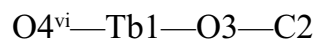 & $-21.7(2)$ \\
\hline $\mathrm{O} 2^{\mathrm{ii}}-\mathrm{Tb} 1-\mathrm{O} 1-\mathrm{Tb} 1^{\mathrm{viii}}$ & 180.0 & $\mathrm{O} 4^{\mathrm{v}}-\mathrm{Tb} 1-\mathrm{O} 3-\mathrm{C} 2$ & $119.5(2)$ \\
\hline 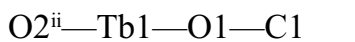 & 0.0 & $\mathrm{O} 4^{\mathrm{vi}}-\mathrm{Tb} 1-\mathrm{O} 3-\mathrm{C} 2$ & $148.72(19)$ \\
\hline
\end{tabular}

Symmetry codes: (i) $x-1 / 2, y,-z+3 / 2$; (ii) $x-1 / 2, y,-z+1 / 2$; (iii) $x,-y+3 / 2, z$; (iv) $-x,-y+1,-z+1$; (v) $-x+1 / 2,-y+1, z+1 / 2$; (vi) $-x, y+1 / 2,-z+1$; (vii) $-x+1 / 2, y+1 / 2, z+1 / 2$; (viii) $x+1 / 2, y,-z+3 / 2$; (ix) $x+1 / 2, y,-z+1 / 2$; (x) $-x+1 / 2,-y+1, z-1 / 2$.

Hydrogen-bond geometry $\left(A,{ }^{\circ}\right)$

\begin{tabular}{lllll}
\hline$D-\mathrm{H} \cdots A$ & $D-\mathrm{H}$ & $\mathrm{H} \cdots A$ & $D \cdots A$ & $D-\mathrm{H} \cdots A$ \\
\hline $\mathrm{C} 1-\mathrm{H} 1 \cdots \mathrm{O} 2^{\mathrm{xi}}$ & 0.93 & 2.15 & $3.051(5)$ & 164 \\
\hline
\end{tabular}

Symmetry code: (xi) $x+1 / 2,-y+3 / 2,-z+1 / 2$. 